\title{
BRCA1/2 mutations and FMR1 alleles are randomly distributed: a case control study
}

\section{This article has been corrected since online publication and a corrigendum also appears in this issue.}

\author{
Efrat Dagan ${ }^{1,2,7}$, Yoram Cohen ${ }^{3,4,7}$, Adi Mory ${ }^{1,5}$, Vardit Adir ${ }^{6}$, Zvi Borochowitz $^{5,6}$, Hila Raanani ${ }^{3}$, \\ Alina Kurolap ${ }^{1,5}$, Svetlana Melikhan-Revzin ${ }^{1,5}$, Dror Meirow ${ }^{3,4}$ and Ruth Gershoni-Baruch ${ }^{\star, 1,5}$
}

BRCA mutation carriers were reported to display a skewed distribution of $F M R 1$ genotypes, predominantly within the low normal range (CGG repeat number $<26$ ). This observation led to the interpretation that $B R C A 1 / 2$ mutations are embryo-lethal, unless rescued by 'low FMR1 alleles'. We undertook to re-explore the distribution of FMR1 alleles subdivided into low, normal and high ( $<26,26-34$, and $>34$ CGG repeats, respectively) subgenotypes, on a cohort of 125 Ashkenazi women, carriers of a BRCA1/2 founder mutation. Ashkenazi healthy females $(n=368)$, tested in the frame of the Israeli screening population program, served as controls. BRCA1/2 carriers and controls demonstrated a comparable and non-skewed FMR 1 subgenotype distribution. Taken together, using a homogeneous ethnic group of Ashkenazi BRCA1/2 mutation carriers, we could not confirm the reported association between FMR 1 low genotypes and BRCA1/2 mutations. The notion that BRCA1/2 mutations are embryo-lethal unless rescued by the low FMR1 subgenotypes is hereby refuted.

European Journal of Human Genetics (2014) 22, 277-279; doi:10.1038/ejhg.2013.281; published online 27 November 2013

Keywords: FMR1 subgenotypes; CGG repeats; BRCA1/2

\section{INTRODUCTION}

The fragile X mental retardation 1 (FMR1) gene, located on the long arm of the $\mathrm{X}$ chromosome, contains a repetitive CGGn trinucleotide segment in the $5^{\prime}$ UTR region of the gene NG_007529.1:g.5061CGG (5_55). Most individuals demonstrate 29-30 CGG triple repeats, the normal range being defined at 5-55 repeats (median $n=30$ ). Expansion of the CGG segment to the so-called pre-mutation range (approximately g.5061CGG (55_200)) is associated with neuropsychiatric risks and primary ovarian insufficiency. ${ }^{1}$ The full mutation range g.5061CGG $(>200)$ instigates gene inactivation and loss of FMR1 protein, thereby causing fragile $\mathrm{X}$ syndrome, an $\mathrm{X}$-linked condition, the leading cause of inherited intellectual disability in humans. ${ }^{2,3}$

Gleicher et $a l^{4-6}$ have constructed a 'private' classification of subgenotypes within the normal FMR1 range (up to 55 repeats). As such, they labeled alleles of 26-34 CGG repeats g.5061CGG(26_34) as 'normal', alleles of less than 26 repeats as 'low range' g.5061CGG(5_25) and those of more than 34 repeats as 'high range' g.5061CGG(35_55). Repeats within the median range g.5061CGG(29_30) correspond allegedly with the switching point between positive and negative message and peak translation of the gene product of FMR1.4-7 Individuals were then defined as 'normal' if both alleles were in the 'normal' range, as 'heterozygous' if one allele was outside the 'normal' range and as homozygous if both alleles were outside of the range. These genotypes were further subdivided based on whether FMR1 alleles were above (high) or below (low) the normal range. ${ }^{4-7}$
This classification was used to show that young women who have 'low or high' CGG counts, whether heterozygous or homozygous, have diminished ovarian reserve. ${ }^{6,7}$ The 29-30 CGG repeat range appears reflective of normal ovarian reserve, with higher and lower counts denoting similar risks towards premature ovarian senescence. $^{4-7}$ Ultimately, the low range allele seems to be associated with premature ovarian aging, primary ovarian insufficiency, occult primary insufficiency, premature menopause, diminished ovarian reserve, and deficient follicle recruitment and IVF outcomes. ${ }^{4-7}$

Other than this, carriers of BRCA1/2 mutations, with breast cancer, were equally reported to endure primary ovarian insufficiency. ${ }^{8}$ BRCA1/2 mutations and FMR1 genotypes, whether low-normal or in the permutation range, were independently reported to be associated with prematurely diminished ovarian reserve. ${ }^{8}$ To test whether the observed effects of BRCA1/2 and FMR1 on ovaries were interrelated, Weghofer et al recently studied 99 women with BRCA1/2 mutations and showed that they preponderantly demonstrated the low FMR1 genotype ( $<26$ CGG repeats) compared with the distribution displayed by a control population of 410 infertile women. ${ }^{9}$ The authors argue that the plausible explanation for such a skewed distribution is that human BRCA1/2 mutations are likely embryo-lethal unless rescued by a low FMR1 subgenotype. ${ }^{9}$ They conclude that women with BRCA1/2-associated breast cancer most likely carry the low FMR1 subgenotypes and that the reported risk towards prematurely diminished ovarian reserve is FMR1-mediated.

\footnotetext{
${ }^{1}$ Institute of Human Genetics, Rambam Health Care Campus, Haifa, Israel; ${ }^{2}$ Department of Nursing, University of Haifa, Haifa, Israel; ${ }^{3}$ IVF Unit, Division of Obstetrics and Gynecology, Chaim Sheba Medical Center, Tel Hashomer, Tel Aviv, Israel; ${ }^{4}$ Sackler School of Medicine, Tel Aviv University, Tel Aviv, Israel; ${ }^{5}$ The Ruth and Bruce Rappaport Faculty of Medicine, Technion Institute of Technology, Haifa, Israel; ${ }^{6}$ The Simon Winter Institute for Human Genetics, Bnai-Zion Medical Center, Haifa, Israel

${ }^{*}$ Correspondence: Professor R Gershoni-Baruch, Institute of Human Genetics, Rambam Health Care Campus and Ruth and Bruce Rappaport Faculty of Medicine, TechnionIsrael Institute, Rambam Medical Center, PO Box 9602, Haifa 31096, Israel. Tel: +972 4 8542456; Fax: +972 4 8543029; E-mail: rgershoni@rambam.health.gov.il ${ }^{7}$ These authors contributed equally to this work.

Received 21 August 2013; revised 30 October 2013; accepted 4 November 2013; published online 27 November 2013
} 
Using this data they generated a hypothesis maintaining that women with low alleles are expected to carry $B R C A 1 / 2$ mutations whereas those carrying normal or high CGGn FMR1 alleles are expected to be $B R C A 1 / 2$ mutation free. ${ }^{9}$

In our Ashkenazi Jewish population, which is quite homogeneous, the prevalence of $B R C A 1 / 2$ founder mutations, namely c.66_ 67delAG (185delAG) and c.5263_5264insC (5382insC) in BRCA1 (NM_007294.3) and c.5946delT (6174delT) in BRCA2 (NM_000059.3), reaches 2.5\%. With all of the above in mind, we undertook to re-explore the distribution of FMR1 subgenotypes, in a cohort of Ashkenazi Jewish females, all carriers of one of the three $B R C A 1 / 2$ founder mutations. ${ }^{10}$

\section{MATERIALS AND METHOD}

\section{Study population}

The study was approved by the Institutional Review Board, at Rambam Health Care Campus, Haifa, Israel. Patients, at time of initial consultation, signed an informed consent, which allows for review of medical records for research purposes as long as confidentiality of the medical record is maintained. All archive files at our Oncogenetic Hereditary Breast-Ovarian Clinic were reviewed in order to identify a study cohort consisting of Ashkenazi women who carried a BRCA1/2 mutation, whether affected $(n=97)$ or non-affected $(n=28)$ by cancer. BRCA1/2 carriers were counseled and tested at our Oncogenetic Clinic between the years 1995 and 2012. Ashkenazi healthy female individuals $(n=368)$, with no family history of developmental impairment, were screened for fragile X syndrome, at the Bnai-Zion Medical Center, Haifa, Israel in the frame of the Israeli screening population program, and selected to have less than 55 CGG repeats, served as a control group. Among the BRCA1/2 carriers: 61 and 19 carried the $185 \mathrm{del} A \mathrm{G}$ and the $5382 \mathrm{ins} \mathrm{C}$ mutation in BRCA1, respectively; 46 had the 6174delT mutation in BRCA2 and one participant had both a 185delAG and a 6174delT mutation. The BRCA1/2 carrier group includes: 97 patients, of whom 66 had breast cancer, 21 had ovarian cancer and 10 had both breast and ovarian cancer; and 25 carrier females with no history of cancer. FMR1 CGGn repeats were tested in the 125 participants from the study group.

\section{Genotyping}

FMR1 alleles. FMR1 testing was performed by PCR amplification using previously described primers ${ }^{11}$ with the GC-RICH PCR System (Roche Applied Science, Penzberg, Germany). Size fragment analysis was carried out by capillary electrophoresis in an ABI PRISM 3100 automatic sequencer (Applied Biosystems, Foster City, CA, USA). This methodology detects only alleles up to a maximum of 70 CGG repeats.

FMR1 data were reported for both alleles as CGGn repeats and as follows: normal range (CGG $n=26-34$ ), low range (less than 26 repeats), high range (between 34 and 55 repeats) and converted into subgenotypes as follows: homozygous (normal, low or high) and heterozygous (normal/low, normal/ high or high/low).

BRCA1/2 ashkenazi mutations. The three predominant founder Ashkenazi mutation, namely, 185delAG and 5382insC in BRCA1; and 6174delT in $B R C A 2$, are routinely tested in breast and/or ovarian cancer patients and family members at risk, at our oncogenetic diagnostic service at Rambam Health Care Campus, as from 1995, using RFLPs as previously described. ${ }^{12,13}$
Statistics. Proportions of FMR1 subgenotypes were compared among groups using cross-tabulations and calculations of Chi-square and $t$-test statistical test when appropriate at 5\% significance level. All statistical calculations were performed using SPSS 19.0 version IBM (Armonk, NY, USA).

\section{RESULTS}

The distribution of FMR1 subgenotypes among BRCA1/2 carriers and healthy controls was comparable (Table 1) and as follows: homozygous normal $68.0 \%(n=85)$ and $55.7 \%(n=205)$, respectively; heterozygous normal/low $22.4 \% \quad(n=28)$ and $26.4 \% \quad(n=97)$, respectively; heterozygous normal/high $7.2 \%(n=9)$ and $11.7 \%$ $(n=43)$, respectively; and heterozygous high/low $1.6 \%(n=2)$ and $3.8 \%(n=14)$, respectively.

Table 2 describes the clinical characteristics of 125 BRCA1/2 mutation carriers related to FMR1 subgenotypes. It shows that $B R C A 1 / 2$ carriers, with at least one low allele, do not differ from those with normal or high alleles as regards morbidity, age at cancer

\section{Table 2 Clinical profiles of $125 B R C A 1 / 2$ mutation carriers related to} FMR1 subgenotypes

\begin{tabular}{|c|c|c|c|}
\hline Clinical profile & $\begin{array}{l}\text { Normal or high } \\
\text { allele, } \mathrm{n}=94\end{array}$ & $\begin{array}{c}\text { At least one low } \\
\text { allele, } \mathrm{n}=31\end{array}$ & $\begin{array}{l}\text { Significant test } \\
\text { (P-value) }\end{array}$ \\
\hline \multicolumn{4}{|c|}{ Site of primary cancer $(n, \%)$} \\
\hline Breast $(n=72)$ & $52(55.3)$ & $20(64.5)$ & \\
\hline Ovary $(n=25)$ & $20(21.3)$ & $5(16.1)$ & \\
\hline Asymptomatic $(n=28)$ & $22(23.4)$ & $6(19.4)$ & \\
\hline Total & $94(75.2)$ & $31(24.8)$ & 0.663 \\
\hline \multicolumn{4}{|l|}{ Age of onset $(M \pm S D)$} \\
\hline Breast cancer & $44.65 \pm 7.62$ & $42.7 \pm 6.71$ & 0.318 \\
\hline Ovarian cancer & $55.25 \pm 11.25$ & $49.0 \pm 7.2$ & 0.253 \\
\hline Asymptomatic & $44.91 \pm 10.86$ & $44.3 \pm 13.7$ & 0.914 \\
\hline \multicolumn{4}{|l|}{ Mutation type ( $n, \%)$} \\
\hline 185delAG & $47(50.0)$ & $14(45.2)$ & \\
\hline 5382insC & $12(12.8)$ & $6(19.4)$ & \\
\hline 6174delT & $35(37.2)$ & $10(32.3)$ & \\
\hline 5382insC/6174delT & - & $1(3.2)$ & \\
\hline Total & 94 & 31 & 0.261 \\
\hline BRCA1 & $59(62.8)$ & $20(66.7)$ & \\
\hline BRCA2 & $35(37.2)$ & $10(33.3)$ & 0.699 \\
\hline \multicolumn{4}{|l|}{ Family history (n, \%) } \\
\hline 1st or 2nd BC-OC-PC & $79(84.0)$ & $27(87.1)$ & \\
\hline Other than BC-OC- PC & $10(10.6)$ & $3(9.7)$ & \\
\hline None & $5(5.3)$ & $1(3.2)$ & 0.428 \\
\hline
\end{tabular}

Abbreviations: $\mathrm{BC}$, breast cancer; OC, ovarian cancer; $\mathrm{PC}$, pancreatic cancer.

$F M R 1$ normal or high CGG repeats compared with at least one low CGG repeats in relation to clinical characteristics of the 125 BRCA1/2 mutation carriers.

Table 1 FMR 1 subgenotype distribution in 125 BRCA1/2 carriers and 368 healthy controls

\begin{tabular}{|c|c|c|c|c|c|c|c|c|c|}
\hline Participants & $\begin{array}{c}\text { Normal/normal } \\
\text { alleles }\end{array}$ & $\begin{array}{c}\text { Normal/high } \\
\text { alleles }\end{array}$ & $\begin{array}{l}\text { High/high } \\
\text { alleles }\end{array}$ & $\begin{array}{c}\text { Total normal or high } \\
\text { allele }\end{array}$ & $\begin{array}{l}\text { Low/high } \\
\text { alleles }\end{array}$ & $\begin{array}{c}\text { Low/normal } \\
\text { alleles }\end{array}$ & $\begin{array}{l}\text { Low/low } \\
\text { alleles }\end{array}$ & $\begin{array}{c}\text { Total with at least one } \\
\text { low allele }\end{array}$ & Total \\
\hline BRCA1/2 carriers $(n, \%)$ & 85 & 9 & - & $94(75.2)$ & 2 & 28 & 1 & $31(24.8)$ & 125 \\
\hline Healthy controls ( $n, \%)$ & 205 & 43 & 4 & $252(68.5)$ & 14 & 97 & 5 & $116(31.5)$ & 368 \\
\hline Total $(n, \%)$ & 290 & 52 & 4 & $346(70.2)$ & 16 & 125 & 6 & $147(29.8)$ & 493 \\
\hline
\end{tabular}

FMR1 subgenotype includes: low alleles $<26$ CGG repeats, normal alleles between 26 and 34 CGG repeats and high alleles $>34$ CGG repeats. 
Table 3 Comparison between FMR1 subgenotypes of 125 BRCA1/2 carriers and 368 healthy controls

\begin{tabular}{lcccc}
\hline & $\begin{array}{c}\text { Normal or high } \\
\text { allele, } \mathrm{n}=346\end{array}$ & $\begin{array}{c}\text { At least one low } \\
\text { allele, } \mathrm{n}=147\end{array}$ & $\begin{array}{c}\text { Total, } \\
\mathrm{n}=493\end{array}$ & $\begin{array}{c}\text { Significant } \\
\text { test }\end{array}$ \\
\hline BRCA1/2 carriers & $94(75.2)$ & $31(24.8)$ & $125(25.4)$ & \\
Controls & $252(68.5)$ & $116(31.5)$ & $368(74.6)$ & $P=0.156$ \\
\hline
\end{tabular}

FMR1 normal or high CGG repeats compared with at least one low CGG repeats in the BRCA1/2 mutation carriers and controls.

onset, type of $B R C A 1 / 2$ mutation and family history of breastovarian-pancreatic cancer. Most BRCA1/2 carriers, diagnosed with breast cancer, harbored preferentially the 185delAG mutation and had a family history for hereditary breast-ovarian-pancreatic syndrome, irrespective of whether they had normal-high alleles or at least one low allele.

Table 3 details the partition of BRCA1/2 mutations and FMR1 subgenotypes in study and control groups. Only $24.8 \%(n=31)$ of $B R C A 1 / 2$ carriers exhibited one low allele comparable to $31.5 \%$ $(n=116)$ of controls $(P=0.156)$.

\section{DISCUSSION}

Diminished ovarian reserve has been independently reported in BRCA1/2 mutation carriers and in women carrying low FMR1 subgenotypes. $^{4-8}$ Weghofer et al hypothesized that the reported association of BRCA1/2 mutations with premature ovarian failure is FMR1-mediated and have lately unequivocally demonstrated that $B R C A 1 / 2$ mutations are almost exclusively associated with the low FMR1 allele. $^{9}$

This observation was challenged by exploring the distribution of FMR1 subgenotypes in a cohort of Ashkenazi women, carriers of a $B R C A 1 / 2$ founder mutation. We found that the FMR1 subgenotypes were equally distributed among our BRCA1/2 carriers of Ashkenazi descent compared with that observed in a control group from the same ethnic background.

Taken together, using a homogeneous group of Ashkenazi BRCA1/2 mutation carriers, we could not confirm the reported association between the low FMR1 subgenotype and BRCA1/2 mutations. The notion that $B R C A 1 / 2$ mutations are embryo-lethal unless rescued by low FMR1 subgenotypes is hereby refuted. BRCA1/2 mutations were reported to be embryo-lethal only if affecting both alleles. ${ }^{14}$ In addition, an international study conducted by us and colleagues, on $2828 B R C A 1 / 2$ carriers, asserts that BRCA1/2 mutation is not a risk factor for spontaneous abortions. ${ }^{15}$ The high prevalence of $B R C A 1 / 2$ founder mutations in the Ashkenazi population does not favor the assumption that $B R C A 1 / 2$ mutation is embryo-lethal.

Lately, Brandao et $a l^{16}$ and Ricci et al ${ }^{17}$ have independently shown that the distribution of the FMR1 subgenotypes in $B R C A$ mutation carriers compares well with that obtained from non carriers, concluding that $B R C A 1 / 2$ mutations are not associated with FMR1 low subgenotypes.

Our case control study has been conducted on relatively large cohorts, from a defined homogenous population, that harbors a limited number of BRCA1/2 founder mutations. We can thus safely conclude that the observation asserting that women with breast cancer, carriers of a $B R C A 1 / 2$ mutation, harbor preferentially a low FMR1 allele, is invalid.

\section{CONFLICT OF INTEREST}

The authors declare no conflict of interest.

\section{ACKNOWLEDGEMENTS}

We acknowledge the Israeli Jack Craps foundation for funding this research.

1 Sullivan SD, Welt C, Sherman S: FMR1 and the continuum of primary ovarian insufficiency. Semin Reprod Med 2011; 29: 299-307.

2 Abrams L, Cronister A, Brown WT et al: Newborn, carrier, and early childhood screening recommendations for fragile X. Pediatrics 2012; 130: 1126-1135.

3 Gallagher A, Hallahan B: Fragile X-associated disorders: a clinical overview. J Neurol 2012; 259: 401-413.

4 Gleicher N, Weghofer A, Barad DH: A pilot study of premature ovarian senescence. I. Correlation of triple CGG repeats on the FMR1 gene to ovarian reserve parameters FSH and anti-Müllerian hormone. Fertil Steril 2009a; 91: 1700-1706.

5 Gleicher N, Weghofer A, Oktay K, Barad D: Relevance of triple CGG repeats in the FMR1 gene to ovarian reserve. Reprod Biomed Online 2009b; 19: 385-390.

6 Gleicher N, Weghofer A, Oktay K, Barad DH: Correlation of triple repeats on the FMR1 (fragile X) gene to ovarian reserve: a new infertility test? Acta Obstet Gynecol Scand 2009c; 8: 1024-1030.

7 Gleicher N, Weghofer A, Barad DH: Ovarian reserve determinations suggest new function of FMR1 (fragile X gene) in regulating ovarian ageing. Reprod Biomed Online 2010; 20: 768-775.

8 Oktay K, Kim JY, Barad D, Babayev SN: Association of BRCA1 mutations with occult primary ovarian insufficiency: a possible explanation for the link between infertility and breast/ovarian cancer risks. J Clin Oncol 2010; 28: 240-244.

9 Weghofer A, Tea MK, Barad DH et al: BRCA1/2 Mutations Appear Embryo-Lethal Unless Rescued by Low (CGG n,26) FMR1 Sub-Genotypes: Explanation for the "BRCA Paradox"? PLoS One 2012; 7: e44753.

10 King MC, Marks JH, Mandell JB: New York Breast Cancer Study Group. Breast and ovarian cancer risks due to inherited mutations in BRCA1 and BRCA2. Science 2003; 302: 643-646.

11 Mornet E, Chateau C, Hirst MC et al: Analysis of germline variation at the FMR1 CGG repeat shows variation in the normal-premutated borderline range. Hum Mol Genet 1996; 5: 821-825.

12 Gershoni-Baruch R, Dagan E, Fried G et al: BRCA1 and BRCA2 founder mutations in patients with bilateral breast cancer. Eur J Hum Genet 1999; 7: 833-836.

13 Rohlfs EM, Learning WG, Friedman KJ et al: Direct detection of mutations in the breast and ovarian cancer susceptibility gene BRCA1 by PCR-mediated site-directed mutagenesis. Clin Chem 1997; 43: 24-29.

14 Konishi H, Mohseni M, Tamaki A et al: Mutation of a single allele of the cancer susceptibility gene BRCA1 leads to genomic instability in human breast epithelial cells. Proc Natl Acad Sci USA 2011; 108: 17773-17778.

15 Friedman E, Kotsopoulos J, Lubinski J et al: Hereditary Breast Cancer Clinical Study Group. Spontaneous and therapeutic abortions and the risk of breast cancer among BRCA mutation carriers. Breast Cancer Res 2006; 8: R15.

16 Brandão RD, van Roozendaal K, Tserpelis D, Blok MJ: FMR1 low sub-genotype does not rescue BRCA1/2-mutated human embryos and does not explain primary ovarian insufficiency among BRCA1/2-carriers. Hum Reprod 2013; 28: 2308-2011.

17 Ricci MT, Pennese L, Gismondi V et al: The FMR1 CGG repeat test is not a candidate prescreening tool for identifying women with a high probability of being carriers of BRCA mutations. Eur J Hum Genet 2014; 22: 280-282. 